\title{
Article \\ Trace Element Analysis in Whole Blood and Plasma for Reference Levels in a Selected Queensland Population, Australia
}

\author{
Tatiana Komarova ${ }^{1}$, Daniel McKeating ${ }^{2}$, Anthony V. Perkins ${ }^{2}$ (1) and Ujang Tinggi ${ }^{1, *}$ \\ 1 Queensland Health Forensic and Scientific Services, Coopers Plains, QLD 4108, Australia; \\ tatiana.komarova@health.qld.gov.au \\ 2 School of Medical Sciences, Griffith University, Parklands Drive, Southport, Gold Coast, QLD 4222, Australia; \\ d.mckeating@griffith.edu.au (D.M.); a.perkins@griffith.edu.au (A.V.P.) \\ * Correspondence: ujang.tinggi@health.qld.gov.au
}

Citation: Komarova, T.; McKeating, D.; Perkins, A.V.; Tinggi, U. Trace

Element Analysis in Whole Blood and Plasma for Reference Levels in a Selected Queensland Population, Australia. Int. J. Environ. Res. Public Health 2021, 18, 2652. https:// doi.org/10.3390/ijerph18052652

Academic Editor: Paul B. Tchounwou

Received: 27 January 2021

Accepted: 26 February 2021

Published: 6 March 2021

Publisher's Note: MDPI stays neutral with regard to jurisdictional claims in published maps and institutional affiliations.

Copyright: (c) 2021 by the authors. Licensee MDPI, Basel, Switzerland. This article is an open access article distributed under the terms and conditions of the Creative Commons Attribution (CC BY) license (https:// creativecommons.org/licenses/by/ $4.0 /)$.

\begin{abstract}
The levels of trace elements in whole blood and plasma have been widely used for assessing nutritional status and monitoring exposure and can vary widely in populations from different geographical regions. In this study, whole blood samples $(n=120)$ and plasma samples ( $n=120$ ) were obtained from healthy donors attending the Red Cross Blood Bank (Queensland Red Cross Blood Service), which provided information for age and sex. There were 71 males (age range: 19-73 years) and 49 females (age range: 18-72 years) for whole blood samples, and 59 males (age range: 19-81 years) and 61 females (age range: 19-73 years) for plasma samples. The main aim of the study was to provide information on blood reference levels of 21 trace elements $(\mathrm{Ag}, \mathrm{Al}, \mathrm{As}, \mathrm{Bi}$, $\mathrm{Br}, \mathrm{Cd}, \mathrm{Co}, \mathrm{Cr}, \mathrm{Cu}, \mathrm{Hg}, \mathrm{I}, \mathrm{Mn}, \mathrm{Mo}, \mathrm{Ni}, \mathrm{Pb}, \mathrm{Sb}, \mathrm{Se}, \mathrm{Tl}, \mathrm{U}, \mathrm{V}, \mathrm{Zn}$ ) in Queensland. The study also aimed to assess differences in trace element blood levels between males and females and the effect of age. The trace element levels in blood samples were analysed using inductively coupled plasma mass spectrometry (ICP-MS) and the standard reference materials of Seronorm (Trace Elements Whole Blood) and UTAK (Trace Elements Serum) were used for quality control and assurance. The study found wide variations of trace element levels in whole blood and plasma, and generally the levels were comparable to other countries. No detectable levels were found for $\mathrm{Bi}, \mathrm{Cr}, \mathrm{U}$ and $\mathrm{V}$ in whole blood, but $\mathrm{V}$ levels were found in plasma samples. There were significant differences between males and females for whole blood $\mathrm{Cu}(p<0.001), \mathrm{I}(p=0.009), \mathrm{Tl}(p=0.016)$ and $\mathrm{Zn}(p=0.016)$. Significant differences were also found for plasma $\mathrm{Cu}(p<0.001)$ and Se $(p=0.003)$ between males and females. There were trends of increased levels of blood $\mathrm{Pb}$, Se and $\mathrm{Zn}$ with age. The study has provided further information on a wide range of trace elements in blood as reference levels for Queensland and Australia which are currently lacking.
\end{abstract}

Keywords: trace elements; reference range values; whole blood; plasma; serum; age group

\section{Introduction}

Trace elements are naturally occurring in the Earth's crust. However, a rapid increase in industrial and agricultural activities over the last few decades has contributed greatly to the distribution of trace elements into the environment. These trace elements will accumulate in the food chain and subsequently a major route of exposure to humans. Increased exposure to trace elements, particularly the toxic metals, has caused concern in human populations. The adverse health effects of metals such as mercury and lead which can cause neurotoxicity or kidney disease from cadmium are well documented [1,2]. Some trace elements are essential for humans and deficiency diseases such as impaired thyroid metabolism from low iodine, anaemia from low iron, and cardiomyopathy from low selenium are well documented [3,4]. 
There has been increased concern about high exposure of trace elements in various populations and this has led to many countries regularly monitoring the levels in human blood, urine, hair and toenails [5-10]. Whole blood samples are frequently used to monitor the levels of trace elements for assessing health status. The levels in whole blood tend to indicate recent and long-term exposure of toxic metals such as lead and cadmium. The main route of exposure of metals in humans includes ingestion of food and water, dermal absorption and inhalation of dust emissions. The levels of trace elements can vary significantly in a population and between geographical regions, and this can depend on dietary habits, occupational environment, lifestyles and genetic variations [11,12].

To the best of our knowledge, there has been no recent study investigating a wide range of trace elements in the Queensland population from various regions and among age groups, sex and health status. However, there have been studies on levels of individual elements such as lead in children blood in Mt. Isa residents and selenium in selected Queensland populations of different ages [13-15]. Our work is the first study providing the reference range values of essential and non-essential trace elements in blood and plasma of a selected cohort of adults in Queensland, Australia. The study will fill a current knowledge gap and provide important information for health workers. The data could be used as a guideline for health assessment from toxic element exposure and evaluation of nutritional status from essential trace element deficiency.

The ICP-MS analytical technique is increasingly being used as a method of choice in many laboratories for analysis of a wide range of trace elements (20-50 elements) in various biological sample matrices including blood and plasma [16,17]. The ICP-MS is a powerful analytical tool that allows multi-element determination from the same sample with high analytical sensitivity. However, there are still challenges for analysis of ultra-low trace element levels $(<\mathrm{ng} / \mathrm{L})$ from complex sample matrices which can contain high levels of various elements and may cause elemental interactions and polyatomic interferences during analysis $[18,19]$. There is also an increased interest in developing methods for low sample volume of clinical samples such as blood for trace element analysis [20,21].

The aim of this study was to conduct analysis of trace element levels in blood samples of selected Queensland population using ICP-MS method to provide reference range values for a wide range of trace elements in adults and to determine the differences of trace element levels between age groups and genders.

\section{Materials and Methods}

\subsection{Equipment and Apparatus}

An inductively coupled plasma mass spectrometer Agilent ICP-MS 7700 (Agilent Technologies, Tokyo, Japan) was used for determination of trace elements in plasma and blood samples. The instrument was equipped with Agilent ASX-500 Series ICP-MS Autosampler (Agilent Technologies, Victoria, Australia) and Integrated Sample Introduction System (ISIS) (Agilent Technologies, Victoria, Australia) for sample introduction.

Dispenser (Hamilton Microlab Dispenser 600 series, Merck) was used to add ultrahigh purity deionised water during blanks, standards and Quality Controls preparation and samples dilution. All samples were homogenised on Ratek Roller Mixer (Ratek Instruments, Victoria, Australia) before taking a required volume for analysis. Blood samples were mixed with alkaline solution using Vortex Mixer (Ratek Instruments, Victoria, Australia).

To prevent contamination, all labware such as $10 \mathrm{~mL}$ plastic tubes (Sarstedt Australia, Mawson Lakes, Australia) and caps for blanks (Sarstedt Australia, Mawson Lakes, Australia), standards, samples and Quality Controls preparation were acid-washing by soaking in $10 \%$ Nitric acid for at least $24 \mathrm{~h}$ before use.

Calibrated pipettes (Eppendorf Multipette E3x, Socorex 100-1000 $\mu \mathrm{L}$, Finnpipete 100-1000 $\mu \mathrm{L}$ ) (Sarstedt Australia, Mawson Lakes, Australia) were used during blanks, standards, quality controls and samples preparation procedures. 


\subsection{Reagents}

Reagents for the preparation of alkaline solution: Triton X100 (1\%) (commercially available detergent, Sigma-Aldrich), 2-propanol/isopropanol (AR Grade, Sigma-Aldrich), ammonia solution (25\% Suprapur, Merck), ethylenediaminetetraacetic acid (EDTA, SigmaAldrich), ultrahigh purity deionised water (DI water, $<2 \mathrm{M} \Omega \times \mathrm{cm}$ at $25^{\circ} \mathrm{C}$ ) produced by water treatment system (Aqua Cure, Burscough, UK).

Reagents for the preparation of standards (high-purity standards from Choice Analytical): Multi-Element Stock Standard Solution containing $10 \mathrm{mg} / \mathrm{L}$ of Ag, Al, As, Bi, Cd, Co, $\mathrm{Cr}, \mathrm{Cu}, \mathrm{Mn}, \mathrm{Mo}, \mathrm{Ni}, \mathrm{Pb}, \mathrm{Sb}, \mathrm{Se}, \mathrm{Tl}, \mathrm{V}$ and $\mathrm{Zn}$ and Single Element Stock Standard Solutions of $\mathrm{Hg}(10 \mathrm{mg} / \mathrm{L}), \mathrm{I}(10 \mathrm{mg} / \mathrm{L}), \mathrm{Br}(1000 \mathrm{mg} / \mathrm{L}), \mathrm{Cu}(1000 \mathrm{mg} / \mathrm{L})$ and $\mathrm{Zn}(1000 \mathrm{mg} / \mathrm{L})$

Reagents for the preparation of internal standard solution for on-line addition (highpurity standards from Choice Analytical): scandium (Sc) $(1000 \mathrm{mg} / \mathrm{L})$, rhodium (Rh) (1000 mg/L), tellurium (Te) (1000 mg/L) and iridium (Ir) (1000 mg/L). Extra whole blood and plasma samples supplied by the Blood Bank were used as blank blood and blank plasma solutions for calibration and matric correction.

\subsection{Certified Reference Materials for Quality Control}

The commercially available Certified Reference Materials were used as quality controls (QCs) in the ICP-MS analysis of blood and plasma samples after reconstituting as per manufacturer's instructions: Seronorm Trace Elements Whole Blood L-1 (SRM1) and Seronorm Trace Elements Whole Blood L-2 (SRM2) (Elitech); UTAK Trace Elements Serum Control Normal Range (UTK1) and UTAK Trace Elements Serum Control High Range (UTK2) (PM Separations). Two sets of quality controls of SRM1 and SRM2 for blood samples and UTK1 and UTK2 for plasma samples were determined at the beginning of the run and end of each run of 20 samples.

\subsection{Sample Collection}

The whole blood samples $(n=120)$ collected in EDTA (purple) blood tubes and serum (plasma) samples $(n=120)$ were obtained from the Red Cross blood bank, Queensland, in agreement with the Griffith University (Material Supply Deed N: 18-04QLD-13). In addition, an institutional clearance to analyse human blood was approved by Griffith University (HREC 2016/423) and Mater Medical Research (Mater Misericordia HREC/MML/64703). Samples were donated by healthy subjects who passed the health screening process prior to sample collection at Red Cross. The samples were collected between the period of 4 months from July to November 2018. The samples were then de-identified and the only information provided by the Red Cross blood bank was age and gender of the donors. The summary statistics of blood and plasma samples from the Red Cross blood bank are shown in Table 1.

Table 1. Age and sex of blood bank donors.

\begin{tabular}{|c|c|c|c|c|c|c|c|c|c|}
\hline \multicolumn{5}{|c|}{ Whole Blood Donor Age (Year) } & \multicolumn{5}{|c|}{ Plasma Donor Age (Year) } \\
\hline Sex & Mean & Median & Min & Max & Sex & Mean & Median & Min & $\operatorname{Max}$ \\
\hline $\mathrm{M}(n=71)$ & & & & & $M(n=59)$ & & & & \\
\hline $\mathrm{F}(n=49)$ & & & & & $\mathrm{F}(n=61)$ & & & & \\
\hline $\mathrm{M}$ and $\mathrm{F}$ & 46.3 & 48 & 18 & 73 & $\mathrm{M}$ and $\mathrm{F}$ & 44.6 & 46 & 19 & 81 \\
\hline M & 47.3 & 49 & 19 & 73 & M & 48.4 & 49 & 19 & 81 \\
\hline $\mathrm{F}$ & 44.9 & 45 & 18 & 72 & $\mathrm{~F}$ & 40.9 & 43 & 19 & 73 \\
\hline
\end{tabular}

\subsection{Sample Preparation and Analysis}

All samples were placed on Ratek roller mixer to ensure complete homogenisation of samples. An aliquot of $0.25 \mathrm{~mL}$ of each sample was taken and added into a $10 \mathrm{~mL}$ acid washed tube with $0.5 \mathrm{~mL}$ alkaline solution and made up to $5 \mathrm{~mL}$ final volume (20-fold dilution). The sample solution was placed on Vortex Mixer for thorough mixing and 
decomposition. Alkaline solution was prepared in a $500 \mathrm{~mL}$ plastic bottle by adding EDTA $(5 \mathrm{~g}), 50 \mathrm{~mL}$ ammonia solution, $50 \mathrm{~mL}$ isopropanol and $5 \mathrm{~mL}$ Triton $\mathrm{X}-100$

The analyses of trace element levels in whole blood and plasma samples were conducted at the Inorganic Chemistry laboratory of Queensland Health Forensic and Scientific Services (QHFSS), a NATA (National Association of Testing Authorities) accredited laboratory. The operating conditions of ICP-MS are shown in Table 2.

Table 2. ICP-MS operating conditions.

\begin{tabular}{cc}
\hline RF Power & $1350 \mathrm{~W}$ \\
RF Matching & $1.95 \mathrm{~V}$ \\
Sample Depth & $8 \mathrm{~mm}$ \\
Nebulizer Gas & $0.8 \mathrm{~L} / \mathrm{min}$ \\
Nebulizer Pump & $0.1 \mathrm{rps}$ \\
Dilution Gas & $0.25 \mathrm{~L} / \mathrm{min}$ \\
Spray Chamber Temp. & $2{ }^{\circ} \mathrm{C}$ \\
Peak Pattern & $1 \mathrm{point}$ \\
Replicates & 3 \\
He flow & $4.5 \mathrm{~mL} / \mathrm{min}$ \\
Sweeps/Replicate & 100 \\
\hline
\end{tabular}

\subsection{ICP-MS Analysis and Conditioning Run}

\subsubsection{Calibration Standard Solutions}

Calibration standard solutions were prepared by transferring the corresponding volumes of intermediate standard solutions into $10 \mathrm{~mL}$ tubes. The intermediate standard solutions were prepared from the Multi-Element and Single Element Stock Standard Solutions after making serial dilutions with DI water. All calibration standards containing $1 \mathrm{~mL}$ alkaline solution was made up to $10 \mathrm{~mL}$ final volume. All blood calibration standards were prepared to contain $0.5 \mathrm{~mL}$ Blank Blood. However, for plasma calibration standards, no blank plasma was added. The concentrations of calibration standard solutions ranged from 0.1 to $100 \mu \mathrm{g} / \mathrm{L}$ for $\mathrm{Ag}, \mathrm{Al}, \mathrm{As}, \mathrm{Bi}, \mathrm{Cd}, \mathrm{Co}, \mathrm{Cr}, \mathrm{Mn}, \mathrm{Mo}, \mathrm{Ni}, \mathrm{Pb}, \mathrm{Sb}, \mathrm{Se}, \mathrm{Tl}$ and V, from 0.1 to $1000 \mu \mathrm{g} / \mathrm{L}$ for $\mathrm{Cu}$ and $\mathrm{Zn}$, from 0.1 to $1 \mu \mathrm{g} / \mathrm{L}$ for $\mathrm{Hg}$ and from 5 to $1000 \mu \mathrm{g} / \mathrm{L}$ for I and 50 to $10,000 \mu \mathrm{g} / \mathrm{L}$ for $\mathrm{Br}$.

\subsubsection{Blanks and Calibration Blanks}

At least seven blank solutions were used in each run. They were prepared by adding $1 \mathrm{~mL}$ of alkaline solution to an acid washed $10 \mathrm{~mL}$ tube with $9 \mathrm{~mL}$ DI water. Blood calibration blank was prepared to contain $1 \mathrm{~mL}$ alkaline solution, $0.5 \mathrm{~mL}$ blood blank and $8.5 \mathrm{~mL}$ DI water. Plasma calibration blank did not contain plasma and had $1 \mathrm{~mL}$ alkaline solution and $9.5 \mathrm{~mL}$ DI water.

\subsubsection{Clinwashes Solutions}

The Clinwashes solutions were prepared in a $50 \mathrm{~mL}$ digestion vessel by adding $5 \mathrm{~mL}$ alkaline solution, $2.5 \mathrm{~mL}$ either blank blood or blank plasma and $42.5 \mathrm{~mL}$ DI water. Plasma and blood Clinwashes solutions were used to condition the ICP-MS sample delivery system and sample cone before calibration (blood analyses) or after calibration (plasma analyses) and used as washes to clean the system during ICP-MS runs.

\subsubsection{Internal Standard Solution}

Internal standard solution (for on-line addition) was prepared to contain $0.5 \%$ nitric acid, $0.5 \%$ hydrochloric acid and high purity standards of Sc, Rh, Te and Ir at concentrations $5,0.4,4$ and $0.4 \mathrm{mg} / \mathrm{L}$, respectively. 


\subsubsection{Preparation of Quality Controls and Spikes}

Certified Reference Materials (CRMs) were reconstituted as per manufacturer's instructions. Then, $0.25 \mathrm{~mL}$ of each CRMs was added into a $10 \mathrm{~mL}$ tube with $0.5 \mathrm{~mL}$ alkaline solution and made up to $5 \mathrm{~mL}$ with DI water for a final 20-fold dilution factor.

A spiked sample included in each batch was prepared by spiking the sample with all elements prepared from the intermediate standard solutions with appropriate concentrations for each element. At least one replicate sample was run for every 20 samples.

\subsection{Statistical Analysis}

The data analysis was carried out using GraphPad Prism v 8.4.1 (GraphPad Software, San Diego, CA, USA) and R version 4.0.2 (R Foundation for Statistical Computing Platform, Vienna, Austria). Results are reported as mean (range), median and percentiles range from P2.5 to P97.5. For statistical purposes and analyses, the concentrations of trace elements with levels $<\mathrm{LOR}(\mathrm{mg} / \mathrm{kg})$ were taken as LOD $\mathrm{mg} / \mathrm{kg}$. Others have used LOD $/ 2$ or LOQ $/ 2$ (limit of quantification/2) for data evaluation $[8,18,21]$. The levels of trace elements were non-normally distributed. Therefore, the non-parametric statistics of Mann-Whitney U Test was used for analysis of significant differences between groups (sex, age). Any difference between groups at $p<0.05$ was considered to be statistically significant.

\section{Results}

\subsection{Quality Control and Assurance}

The levels of trace elements in Quality Controls were within the range of the reference values of the Seronorm Trace Elements Whole Blood (Table 3), except for Br and I which were consistently lower for all ICP-MS analysis of blood samples. Concentrations of Ag, $\mathrm{Bi}, \mathrm{Cr}, \mathrm{Ni}$, and $\mathrm{Tl}$ in Seronorm Whole Blood $\mathrm{L}-1$ were below the method detection limits. The levels of trace elements in the UTAK Trace Element Serum Controls were within the range of the reference values. The expected concentrations of $\mathrm{Bi}$, $\mathrm{Tl}$ and $\mathrm{V}$ were below the detection limits of the method.

Table 3. Mean levels of trace elements in the Certified Reference Materials of quality control for whole blood and plasma.

\begin{tabular}{|c|c|c|c|c|c|c|c|c|c|}
\hline \multirow[b]{2}{*}{ Element } & \multirow[b]{2}{*}{ Unit } & \multicolumn{4}{|c|}{ Seronorm Whole Blood QC } & \multicolumn{4}{|c|}{ UTAK Trace Element Serum Control } \\
\hline & & $\begin{array}{l}\text { This } \\
\text { Study }\end{array}$ & Whole Blood L-1 & $\begin{array}{l}\text { This } \\
\text { Study }\end{array}$ & Whole Blood L-2 & $\begin{array}{l}\text { This } \\
\text { Study }\end{array}$ & Normal Range & $\begin{array}{l}\text { This } \\
\text { Study }\end{array}$ & High Range \\
\hline $\mathrm{Ag}$ & $\mu \mathrm{g} / \mathrm{L}$ & $<0.1$ & 0.075 & $<0.1$ & 0.073 & - & - & - & - \\
\hline $\mathrm{Al}$ & $\mu \mathrm{g} / \mathrm{L}$ & - & $11.6(5.8-17.5)$ & - & $68.9(55.0-82.7)$ & 13.0 & $10.9(6.5-15.3)$ & 192 & $\begin{array}{c}201 \\
(161-241)\end{array}$ \\
\hline As & $\mu \mathrm{g} / \mathrm{L}$ & 1.93 & $2.4(1.9-29)$ & 11.6 & $14.1(11.3-17.0)$ & - & - & - & - \\
\hline $\mathrm{Bi}$ & $\mu \mathrm{g} / \mathrm{L}$ & $<0.1$ & - & 5.27 & $5.00(3.99-6.00)$ & $<0.08$ & - & $<0.08$ & - \\
\hline $\mathrm{Br}$ & $\mu \mathrm{g} / \mathrm{L}$ & 714 & 1053 & 693 & 980 & - & - & - & - \\
\hline $\mathrm{Cd}$ & $\mu \mathrm{g} / \mathrm{L}$ & $<0.8$ & $0.28(0.17-0.40)$ & 4.86 & $5.01(4.00-6.02)$ & - & $0.17(0.13-0.21)$ & - & $9.0(7.2-10.8)$ \\
\hline Co & $\mu \mathrm{g} / \mathrm{L}$ & 0.21 & $0.20(0.12-0.28)$ & 5.01 & $5.18(4.13-6.22)$ & 0.27 & - & 0.29 & - \\
\hline $\mathrm{Cr}$ & $\mu \mathrm{g} / \mathrm{L}$ & $<5$ & $0.45(0.27-0.63)$ & 9.4 & $10.7(8.5-12.8)$ & 0.49 & $0.16(0.12-0.20)$ & 2.42 & $\begin{array}{c}2.27 \\
(1.82-2.72)\end{array}$ \\
\hline $\mathrm{Cu}$ & $\mathrm{mg} / \mathrm{L}$ & 0.58 & $0.64(0.51-0.76)$ & 1.17 & $1.34(1.07-1.60)$ & 0.99 & $1.08(0.81-1.35)$ & 2.76 & $3.06(2.4-3.7)$ \\
\hline $\mathrm{Hg}$ & $\mu \mathrm{g} / \mathrm{L}$ & 1.45 & $1.48(1.18-1.77)$ & 17.1 & $17.0(13.6-20.4)$ & - & $\begin{array}{c}1.00(0.01-1.00) \\
-\end{array}$ & - & $\begin{array}{c}0.00(2.4-0.1) \\
-\end{array}$ \\
\hline I & $\mu \mathrm{g} / \mathrm{L}$ & 17.1 & $28.6(22.8-34.3)$ & 74 & $107(86-129)$ & - & - & - & - \\
\hline Mn & $\mu \mathrm{g} / \mathrm{L}$ & 17.3 & $18.4(14.7-22.1)$ & 29.8 & $31.4(25.1-37.7)$ & 0.56 & $0.47(0.35-0.59)$ & 2.47 & $\begin{array}{c}2.51 \\
(2.01-3.01)\end{array}$ \\
\hline Mo & $\mu \mathrm{g} / \mathrm{L}$ & 0.58 & $0.51(0.41-0.61)$ & 4.69 & $5.31(4.24-6.37)$ & 0.92 & - & 0.88 & - \\
\hline $\mathrm{Ni}$ & $\mu \mathrm{g} / \mathrm{L}$ & $<2$ & $1.38(1.10-1.66)$ & 15.1 & $15.9(12.7-19.1)$ & - & - & - & - \\
\hline $\mathrm{Pb}$ & $\mu \mathrm{g} / \mathrm{L}$ & 10.1 & $9.9(7.9-11.9)$ & 324 & $337(269-405)$ & - & - & - & - \\
\hline $\mathrm{Sb}$ & $\mu \mathrm{g} / \mathrm{L}$ & 1.43 & $1.33(1.06-1.60)$ & 25.5 & $25.9(20.7-31.1)$ & - & - & - & - \\
\hline Se & $\mu \mathrm{g} / \mathrm{L}$ & 50 & $60(48-72)$ & 139 & $161(128-193)$ & 110 & $101(76-126)$ & 309 & $\begin{array}{c}281 \\
(225-337)\end{array}$ \\
\hline $\mathrm{Tl}$ & $\mathrm{ng} / \mathrm{L}$ & $<0.06$ & $7(3-11)$ & 10.9 & $10.2(8.1-12.2)$ & $<0.04$ & - & $<0.04$ & - \\
\hline $\mathrm{U}$ & $\mu \mathrm{g} / \mathrm{L}$ & 0.21 & 0.18 & 0.19 & 0.18 & - & - & - & - \\
\hline $\mathrm{V}$ & $\mu \mathrm{g} / \mathrm{L}$ & 0.63 & $0.97(0.58-1.35)$ & 4.02 & $4.98(3.98-5.98)$ & $<0.20$ & - & $<0.20$ & - \\
\hline $\mathrm{Zn}$ & $\mathrm{mg} / \mathrm{L}$ & 4.2 & $(3.4-5.2)$ & 7.2 & $7.1(5.7-8.5)$ & 0.69 & $0.68(0.51-0.85)$ & 2.57 & $\begin{array}{c}2.64 \\
(2.11-3.17)\end{array}$ \\
\hline
\end{tabular}

The recoveries of trace elements from the spiked blood samples ranged from $96 \%$ to $107 \%$ and from $95 \%$ to $108 \%$ for plasma samples. Slightly high recovery of Se $(121 \%)$ was found in spiked plasma samples (Table 4). However, the concentrations of Se in UTK1 and 
UTK2 were within the expected ranges (Table 3). Spike recovery for $\mathrm{Hg}$ in whole blood samples is characterised by wide variation (Table 4). This can be related to the use of a solution with quite low and unstable level of $\mathrm{Hg}(0.5 \mu \mathrm{g} / \mathrm{L})$ as a spike. Concentrations of $\mathrm{Hg}$ found in SRM1 and SRM2 complied with the certified values (Table 3).

Table 4. Spike recoveries of elements from whole blood and plasma.

\begin{tabular}{|c|c|c|c|}
\hline \multirow[t]{2}{*}{ Elements } & \multirow[t]{2}{*}{ Spike Level $(\mu \mathrm{g} / \mathrm{L})$} & $\begin{array}{c}\text { Whole Blood Spike } \\
\text { Recovery (\%) }\end{array}$ & $\begin{array}{l}\text { Plasma Spike } \\
\text { Recovery (\%) }\end{array}$ \\
\hline & & \multicolumn{2}{|c|}{ Mean \pm s.d. $(n=3)$} \\
\hline $\mathrm{Al}$ & 5 & - & $101.0 \pm 3.4$ \\
\hline $\mathrm{Ag}$ & 5 & $100.5 \pm 2.8$ & - \\
\hline As & 5 & $104.0 \pm 5.6$ & - \\
\hline $\mathrm{Bi}$ & 5 & $101.2 \pm 2.1$ & $94.9 \pm 1.8$ \\
\hline $\mathrm{Br}$ & 500 & $98.9 \pm 3.0$ & - \\
\hline $\mathrm{Cd}$ & 5 & $99.5 \pm 2.5$ & - \\
\hline $\mathrm{Co}$ & 5 & $97.9 \pm 2.2$ & $104.3 \pm 1.8$ \\
\hline $\mathrm{Cr}$ & 5 & $96.0 \pm 1.4$ & $96.3 \pm 1.4$ \\
\hline $\mathrm{Cu}$ & 505 & $101.0 \pm 5.0$ & $98.8 \pm 1.5$ \\
\hline $\mathrm{Hg}$ & 0.5 & $120 \pm 47$ & - \\
\hline I & 50 & $99.4 \pm 1.4$ & - \\
\hline $\mathrm{Mn}$ & 5 & $101.1 \pm 6.0$ & $105.4 \pm 3.6$ \\
\hline Mo & 5 & $97.9 \pm 1.8$ & $108.4 \pm 1.0$ \\
\hline $\mathrm{Ni}$ & 5 & $98.5 \pm 3.1$ & - \\
\hline $\mathrm{Pb}$ & 5 & $107.2 \pm 15.2$ & - \\
\hline $\mathrm{Sb}$ & 5 & $96.7 \pm 1.3$ & - \\
\hline Se & 5 & $100.5 \pm 5.0$ & $121.1 \pm 6.2$ \\
\hline $\mathrm{Tl}$ & 5 & $97.6 \pm 2.0$ & $95.9 \pm 1.8$ \\
\hline U & 5 & $98.2 \pm 2.9$ & - \\
\hline $\mathrm{V}$ & 5 & $99.9 \pm 2.9$ & $99.1 \pm 0.9$ \\
\hline $\mathrm{Zn}$ & 505 & $104.3 \pm 10.5$ & $103.7 \pm 1.4$ \\
\hline
\end{tabular}

Our Inorganic Chemistry laboratory has also regularly participated in Quality Assurance Programs organised by The Royal College of Pathologists of Australasia (RCPAQAP), and our results were within the acceptable ranges for all requested trace metals.

\subsection{Trace Element Levels in Blood}

The levels of trace elements in whole blood and plasma varied widely and the results are summarised in Table 5. The levels of $\mathrm{Bi}, \mathrm{Cr}, \mathrm{U}$ and $\mathrm{V}$ in blood samples were below the limits of detection of the method. The values of trace element limits of detection (LOD) and limits of reporting (LOR) for the method are shown in Table 5 . The LODs were calculated from a series of blank samples $(n=21)$ analysed in different days as three times the standard deviation of the blanks multiplied by sample dilution factor of 20. LORs were calculated as three times LODs.

The trace element levels between females and males in whole blood and plasma samples were evaluated using non-parametric Mann-Whitney U Test for statistical differences. There were significant differences for levels of $\mathrm{Cu}(p<0.001), \mathrm{I}(p=0.009), \mathrm{Tl}(p=0.016)$ and $\mathrm{Zn}(p=0.016)$ in whole blood with higher levels of $\mathrm{Cu}$ and I, but lower levels of $\mathrm{Tl}$ and $\mathrm{Zn}$ in females in comparison to males (Table 6). There were significant differences between sexes for $\mathrm{Cu}(p<0.001)$ and Se $(p=0.003)$ in plasma, with higher Cu level $(1240 \mu \mathrm{g} / \mathrm{L})$ and lower Se level (124 $\mu \mathrm{g} / \mathrm{L})$ in females compared to males (Table 6). 
Table 5. The levels of trace elements in whole blood and plasma including method limit of detection (LOD) and limit of reporting (LOR).

\begin{tabular}{|c|c|c|c|c|c|c|}
\hline \multirow{2}{*}{ Element $(\mu \mathrm{g} / \mathrm{L})$} & \multirow{2}{*}{$\begin{array}{c}\text { Whole Blood }(n=120) \\
\text { Mean (Range) }\end{array}$} & \multirow{2}{*}{$\begin{array}{c}\text { Plasma }(n=120) \\
\text { Mean (Range) }\end{array}$} & \multicolumn{2}{|c|}{ Whole Blood } & \multicolumn{2}{|c|}{ Plasma } \\
\hline & & & LOD & LOR & LOD & LOR \\
\hline $\mathrm{Ag}$ & $0.19(<0.1-0.82)$ & - & 0.04 & 0.1 & - & - \\
\hline $\mathrm{Al}$ & - & $6.9(4-82)$ & - & - & 1.2 & 4.0 \\
\hline As & $2.2(<0.2-41.64)$ & - & 0.06 & 0.2 & - & - \\
\hline $\mathrm{Bi}$ & $<0.1$ & $<0.08$ & 0.04 & 0.1 & 0.02 & 0.08 \\
\hline $\mathrm{Br}$ & $4960(2820-12,200)$ & - & 18 & 52 & - & - \\
\hline $\mathrm{Cd}$ & $0.80(<0.8-0.99)$ & - & 0.2 & 0.8 & - & - \\
\hline $\mathrm{Co}$ & $0.33(<0.2-1.1)$ & $0.47(0.21-1.3)$ & 0.06 & 0.2 & 0.02 & 0.08 \\
\hline $\mathrm{Cr}$ & $<5$ & $<1.7$ & 1.8 & 5.0 & 0.6 & 1.7 \\
\hline $\mathrm{Cu}$ & $840(650-1420)$ & $1100(670-2490)$ & 0.6 & 1.6 & 0.4 & 1.0 \\
\hline $\mathrm{Hg}$ & $2.0(<0.8-9.3)$ & - & 0.2 & 0.8 & - & - \\
\hline $\mathrm{I}$ & $30.1(19.5-82.7)$ & - & 0.4 & 1.5 & - & - \\
\hline $\mathrm{Mn}$ & $9.7(4.54-19.5)$ & $1.0(<1-3.1)$ & 0.2 & 0.8 & 0.4 & 1.0 \\
\hline Mo & $0.49(<0.3-1.7)$ & $0.91(0.26-3.0)$ & 0.2 & 0.3 & 0.08 & 0.2 \\
\hline $\mathrm{Ni}$ & $2.0(<2-4.0)$ & - & 0.8 & 2.0 & - & - \\
\hline $\mathrm{Pb}$ & $13.6(3.8-49.6)$ & - & 0.2 & 0.3 & - & - \\
\hline $\mathrm{Sb}$ & $4.1(2.6-6.2)$ & - & 0.2 & 0.3 & - & - \\
\hline Se & $141(118-224)$ & $130(82-180)$ & 0.6 & 2.0 & 0.6 & 1.6 \\
\hline $\mathrm{Tl}$ & $0.06(<0.06-0.16)$ & $0.11(0.04-0.20)$ & 0.02 & 0.06 & 0.02 & 0.04 \\
\hline $\mathrm{U}$ & $<0.1$ & - & 0.04 & 0.1 & - & - \\
\hline $\mathrm{V}$ & $<0.45$ & $0.2(0.2-0.22)$ & 0.2 & 0.45 & 0.06 & 0.20 \\
\hline $\mathrm{Zn}$ & $6750(4620-9250)$ & $1150(820-1660)$ & 4 & 11.5 & 1.6 & 5.0 \\
\hline
\end{tabular}

${ }^{\text {a }}$ For statistical purposes, the trace element concentrations $<$ LOR $(\mathrm{mg} / \mathrm{kg})$ were taken as LOD $(\mathrm{mg} / \mathrm{kg})$ to determine for mean values.

Table 6. Trace element levels (mean and range) in whole blood and plasma of males and females.

\begin{tabular}{|c|c|c|c|c|c|c|}
\hline \multirow{2}{*}{ Element $(\mu \mathrm{g} / \mathrm{L})$} & \multicolumn{2}{|c|}{ Whole Blood $(n=120)$} & \multirow{2}{*}{$\begin{array}{c}\text { Mann- } \\
\text { Whitney } \\
\text { U Test } \\
\text { Significance }\end{array}$} & \multicolumn{2}{|c|}{ Plasma $(n=120)$} & \multirow{2}{*}{$\begin{array}{c}\text { Mann- } \\
\text { Whitney } \\
\text { U Test } \\
\text { Significance }\end{array}$} \\
\hline & Female $(n=49)$ & Male $(n=71)$ & & Female $(n=61)$ & Male $(n=59)$ & \\
\hline $\mathrm{Ag}$ & $0.20(0.1-0.67)$ & $0.17(0.1-0.82)$ & & - & - & \\
\hline $\mathrm{Al}$ & - & - & & $6.4(<4-21.8)$ & $7.4(<4-81.8)$ & \\
\hline As & $2.7(0.2-42)$ & $1.8(0.2-13.0)$ & & - & - & \\
\hline $\mathrm{Bi}$ & $<0.1$ & $<0.1$ & & $<0.08$ & $<0.08$ & \\
\hline $\mathrm{Br}$ & $\begin{array}{c}5140 \\
(2820-12,180\end{array}$ & $\begin{array}{c}4830 \\
(3120-9050)\end{array}$ & & - & - & \\
\hline $\mathrm{Cd}$ & $0.81(<0.8-0.99)$ & $0.80(<0.8-0.93)$ & & - & - & \\
\hline $\mathrm{Co}$ & $0.37(<0.2-1.1)$ & $0.30(<0.2-0.86)$ & & $0.50(0.22-1.34)$ & $0.44(0.21-1.1)$ & \\
\hline $\mathrm{Cr}$ & $<5$ & $<5$ & & $<1.7$ & $<1.7$ & \\
\hline $\mathrm{Cu}$ & $930(710-1420)$ & 770 (650-950) & $p<0.001$ & $1240(706-2485)$ & $960(672-1403)$ & $p<0.001$ \\
\hline $\mathrm{Hg}$ & $1.8<0.8-9.3)$ & $2.1(<0.8-7.7)$ & & - & - & \\
\hline I & $32.5(20.1-82.7)$ & $28.5(19.5-44.6)$ & $p=0.009$ & - & - & \\
\hline Mn & $10.0(5.7-17.6)$ & $9.6(4.5-19.5)$ & & $1.1(<1-3.1)$ & $1.0(<1-1.3)$ & \\
\hline Mo & $0.51(<0.3-1.7)$ & $0.47(<0.3-1.2)$ & & $0.85(0.27-2.67)$ & $0.96(0.26-3.0)$ & \\
\hline $\mathrm{Ni}$ & $2.0(<2-4.0)$ & $<2$ & & - & - & \\
\hline $\mathrm{Pb}$ & $13.2(3.8-49.6)$ & $13.9(4.9-45.0)$ & & - & - & \\
\hline $\mathrm{Sb}$ & $4.0(2.8-5.9)$ & $4.1(2.6-6.2)$ & & - & - & \\
\hline Se & $140(122-203)$ & $142(118-224)$ & & $124(82-179)$ & $130(101-161)$ & $p=0.003$ \\
\hline $\mathrm{Tl}$ & $\begin{array}{c}0.061 \\
(<0.06-0.10)\end{array}$ & $\begin{array}{c}0.07 \\
(<0.06-0.16)\end{array}$ & $p=0.016$ & $0.11(0.05-0.20)$ & $0.10(0.04-0.20)$ & \\
\hline $\mathrm{U}$ & $<0.1$ & $<0.1$ & & - & - & \\
\hline $\mathrm{V}$ & $<0.45$ & $<0.45$ & & $0.20(<0.2-0.22)$ & $0.20(<0.2-0.22)$ & \\
\hline $\mathrm{Zn}$ & $\begin{array}{c}6540 \\
(4940-8390)\end{array}$ & $\begin{array}{c}6900 \\
(4620-9250)\end{array}$ & $p=0.016$ & $1140(850-1660)$ & 1160 (819-1496) & \\
\hline
\end{tabular}

Significant differences were found between female and male for whole blood $\mathrm{Cu}, \mathrm{I}, \mathrm{Tl}$ and $\mathrm{Zn}$, and for plasma $\mathrm{Cu}$ and $\mathrm{Se}$. 
The median age of the Australian population is approximately 40 years old, and this median age is used to divide the participants into groups of under 40 years and over 40 years of age [22]. The results showed significant differences between groups for whole blood levels of $\mathrm{Ag}(p=0.034), \mathrm{Hg}(p=0.031), \mathrm{Pb}(p=0.003)$, Se $(p=0.026)$ and $\mathrm{Tl}(p=<0.001)$ (Table 7). A generalised linear regression was used to evaluate correlations and trends between blood trace element levels and the age of the participants (Figure S1).

Table 7. Trace element levels in blood for under and over 40 years old.

\begin{tabular}{|c|c|c|c|c|c|c|}
\hline \multirow{2}{*}{ Element ( $\mu \mathrm{g} / \mathrm{L})$} & \multicolumn{2}{|c|}{ Whole Blood } & \multirow{2}{*}{$\begin{array}{c}\text { Mann-Whitney } \\
\text { U Test } \\
\text { Significance }\end{array}$} & \multicolumn{2}{|c|}{ Plasma } & \multirow{2}{*}{$\begin{array}{c}\text { Mann-Whitney } \\
\text { U Test } \\
\text { Significance }\end{array}$} \\
\hline & $\begin{array}{l}\text { Under } 40 y \\
\quad(n=43)\end{array}$ & Over 40 y $(n=77)$ & & $\begin{array}{c}\text { Under } 40 \mathrm{y} \\
(n=45)\end{array}$ & Over 40 y $(n=75)$ & \\
\hline $\mathrm{Ag}$ & $0.17(<0.1-0.65)$ & $0.20(<0.1-0.82)$ & $p=0.034$ & - & - & \\
\hline $\mathrm{Al}$ & - & - & & $6.2(<4-9.6)$ & $7.4(<4-81.8)$ & \\
\hline As & $1.53(<0.2-13.4)$ & $2.51(<0.2-41.6)$ & & - & - & \\
\hline $\mathrm{Bi}$ & $<0.1$ & $<0.1$ & & $<0.08$ & $<0.08$ & \\
\hline $\mathrm{Br}$ & $4740(2820-9050)$ & $\begin{array}{c}5080 \\
(3110-12,180)\end{array}$ & & - & - & \\
\hline $\mathrm{Cd}$ & $0.80(<0.8-0.85)$ & $0.81(<0.8-0.99)$ & & - & - & \\
\hline Co & $0.31(<0.2-0.86)$ & $0.34(<0.2-1.1)$ & & $0.47(0.21-1.2)$ & $0.47(0.21-1.34)$ & \\
\hline $\mathrm{Cr}$ & $<5$ & $<5$ & & $<1.7$ & $<1.7$ & \\
\hline $\mathrm{Cu}$ & 837 (649-1390) & $840(660-1420)$ & & $1223(706-2490)$ & 1030 (672-1963) & \\
\hline $\mathrm{Hg}$ & $1.52(<0.8-6.71)$ & $2.2355(<0.8-9.3)$ & $p=0.031$ & - & - & \\
\hline I & $30.0(19.8-42.7)$ & $30.3(19.5-82.7)$ & & - & - & \\
\hline Mn & $10.2(5.3-19.5)$ & 9.5 (4.5-19.3) & & $1.0(<1-1.3)$ & $1.1(<1-3.1)$ & \\
\hline Mo & $0.50(0.3-1.7)$ & $0.48(0.3-1.2)$ & & $0.94(0.29-2.67)$ & $0.89(0.26-3.0)$ & \\
\hline $\mathrm{Ni}$ & $2.0(<2-4.0)$ & $<2$ & & - & - & \\
\hline $\mathrm{Pb}$ & $10.9(4.8-31.4)$ & $15.1(3.8-49.6)$ & $p=0.003$ & - & - & \\
\hline $\mathrm{Sb}$ & $4.0(2.8-5.1)$ & $4.1(2.6-6.2)$ & & - & - & \\
\hline Se & 137 (119-156) & $144(118-224)$ & $p=0.026$ & $124(96-161)$ & $129(82-179)$ & $p=0.013$ \\
\hline $\mathrm{Tl}$ & $0.07(<0.06-0.11)$ & $0.062(<0.06-0.16)$ & $p<0.001$ & $0.11(0.05-0.20)$ & $0.10(0.04-0.16)$ & \\
\hline $\mathrm{U}$ & $<0.1$ & $<0.1$ & & - & - & \\
\hline $\mathrm{V}$ & $<0.45$ & $<0.45$ & & $0.20(<0.20-0.20)$ & $0.20(<0.20-0.22)$ & \\
\hline $\mathrm{Zn}$ & $6590(4620-8650)$ & 6840 (4910-9250) & & $1100(820-1340)$ & $1180(890-1660)$ & $p=0.011$ \\
\hline
\end{tabular}

Significant differences were found between age groups (under and over 40 years) for whole blood $\mathrm{Ag}, \mathrm{Hg}$, $\mathrm{Pb}$, Se and $\mathrm{Tl}$, and for plasma Se and $\mathrm{Zn}$.

There were some correlations between age and blood $\mathrm{Hg}, \mathrm{Pb}$, Se and $\mathrm{Zn}$ which indicated a trend of increased levels of these elements in whole blood with age (Figure S1). Significant correlations were also observed between age and plasma Se and $\mathrm{Cu}$ with increased trend for Se with age and decreased trend for $\mathrm{Cu}$.

The results of whole blood and plasma concentrations were presented as geometric means (GM) for the percentile ranges (Table 8). This would provide meaningful ranges when the impact of any outliers was reduced for percentile ranges. The results in Table 8 also show that trace element blood levels between males and females are generally comparable, except for $\mathrm{Cu}$ and $\mathrm{Br}$ which were relatively higher in females than males.

Table 8. Trace element levels in whole blood and plasma as percentile range for females and males.

\begin{tabular}{ccccccccc}
\hline \multirow{2}{*}{$\begin{array}{c}\text { Element } \\
(\mu \mathrm{g} / \mathrm{L})\end{array}$} & \multicolumn{9}{c}{ Whole Blood Percentile Range } \\
\cline { 2 - 9 } & $\mathbf{2 . 5 - 1 0 \%}$ & $\mathbf{2 5 - 7 5 \%}$ & $\mathbf{9 0 - 9 7 . 5 \%}$ & $\mathbf{G M} \mathbf{~ a}$ & $\mathbf{2 . 5 - 1 0 \%}$ & $\mathbf{2 5 - 7 5 \%}$ & $\mathbf{9 0 - 9 7 . 5 \%}$ & $\mathbf{G M}$ \\
\hline $\mathrm{Ag}$ & $<0.1$ & $0.10-0.25$ & $0.31-0.61$ & 0.17 & $<0.1$ & $<0.1-0.21$ & $0.26-0.44$ & 0.15 \\
$\mathrm{As}$ & $<0.2$ & $0.45-1.7$ & $5.0-13.4$ & 1.0 & $<0.2-0.25$ & $0.46-1.73$ & $3.5-12.1$ & 0.95 \\
$\mathrm{Bi}$ & $<0.1$ & $<0.1$ & $<0.1$ & $<0.1$ & $<0.1$ & $<0.1$ & $<0.1$ & $<0.1$ \\
$\mathrm{Br}$ & $3140-3950$ & $4520-5590$ & $6380-6700$ & 4990 & $3270-3500$ & $4030-5610$ & $5980-7890$ & 4710 \\
$\mathrm{Cd}$ & $<0.8$ & $<0.8$ & $<0.8-0.86$ & 0.81 & $<0.8$ & $<0.8$ & $<0.8$ & $<0.8$ \\
$\mathrm{Co}$ & $<0.2$ & $<0.2-0.46$ & $0.63-0.86$ & 0.33 & $<0.2$ & $<0.2-0.34$ & $0.48-0.57$ & 0.28 \\
$\mathrm{Cr}$ & $<5$ & $<5$ & $<5$ & $<5$ & $<5$ & $<5$ & $<5$ & $<5$ \\
$\mathrm{Cu}$ & $720-750$ & $810-1020$ & $1160-1390$ & 920 & $660-680$ & $730-810$ & $850-940$ & 770 \\
$\mathrm{Hg}$ & $<0.8$ & $<0.81 .9$ & $3.3-6.7$ & 1.4 & $<0.8$ & $<0.8-2.6$ & $4.9-6.8$ & 1.6 \\
\hline
\end{tabular}


Table 8. Cont.

\begin{tabular}{|c|c|c|c|c|c|c|c|c|}
\hline \multirow{3}{*}{$\begin{array}{l}\text { Element } \\
(\mu \mathrm{g} / \mathrm{L})\end{array}$} & \multicolumn{8}{|c|}{ Whole Blood Percentile Range } \\
\hline & \multicolumn{4}{|c|}{ Female $(n=49)$} & \multicolumn{4}{|c|}{ Male $(n=71)$} \\
\hline & $2.5-10 \%$ & $25-75 \%$ & $90-97.5 \%$ & $\mathrm{GM}^{\mathrm{a}}$ & $2.5-10 \%$ & $25-75 \%$ & $90-97.5 \%$ & GM \\
\hline I & $22.6-25.8$ & $26.1-34.0$ & $40.6-60.2$ & 31 & $20.6-22.9$ & $25.2-30.9$ & $34.7-37.8$ & 28.1 \\
\hline Mn & $6.6-7.3$ & $8.0-11.5$ & $13.0-13.9$ & 9.7 & $5.2-6.2$ & $7.0-11.3$ & $13.4-18.7$ & 9.1 \\
\hline Mo & $<0.3$ & $0.35-0.61$ & $0.75-1.1$ & 0.47 & $<0.3$ & $0.3-0.54$ & $0.77-0.98$ & 0.43 \\
\hline $\mathrm{Ni}$ & $<2$ & $<2$ & $<2$ & $<2$ & $<2$ & $<2$ & $<2$ & $<2$ \\
\hline $\mathrm{Pb}$ & $4.5-5.5$ & $8.0-15.3$ & $24.3-33.4$ & 11.2 & $5.4-6.7$ & $9.2-16.5$ & $19.6-33.4$ & 12.5 \\
\hline $\mathrm{Sb}$ & $3.0-3.2$ & $3.4-4.3$ & $4.7-5.0$ & 3.9 & $2.9-3.4$ & $3.7-4.5$ & $5.0-5.8$ & 4.1 \\
\hline Se & $122-124$ & $130-148$ & $157-171$ & 140 & $120-123$ & $131-152$ & $158-167$ & 140 \\
\hline $\mathrm{Tl}$ & $<0.06$ & $<0.06$ & $0.062-0.072$ & 0.061 & $<0.06$ & $<0.06-0.062$ & $0.08-0.10$ & 0.065 \\
\hline $\mathrm{U}$ & $<0.1$ & $<0.1$ & $<0.1$ & $<0.1$ & $<0.1$ & $<0.1$ & $<0.1$ & $<0.1$ \\
\hline $\mathrm{V}$ & $<0.45$ & $<0.45$ & $<0.45$ & $<0.45$ & $<0.45$ & $<0.45$ & $<0.45$ & $<0.45$ \\
\hline $\mathrm{Zn}$ & $5250-5650$ & $5910-7070$ & $7660-8350$ & 6490 & $5120-5740$ & $6270-7480$ & $8140-8570$ & 6840 \\
\hline \multirow{3}{*}{$\begin{array}{c}\text { Element } \\
(\mu \mathrm{g} / \mathrm{L})\end{array}$} & \multicolumn{8}{|c|}{ Plasma Percentile Range } \\
\hline & \multicolumn{4}{|c|}{ Female $(n=61)$} & \multicolumn{4}{|c|}{ Male $(n=59)$} \\
\hline & $2.5-10 \%$ & $25-75 \%$ & $90-97.5 \%$ & $\mathrm{GM}^{\mathrm{a}}$ & $2.5-10 \%$ & $25-75 \%$ & $90-97.5 \%$ & GM \\
\hline $\mathrm{Al}$ & $<4-4.4$ & $5.4-6.8$ & $8.2-10.1$ & 6.2 & $<4$ & $4.7-6.9$ & $8.2-12.0$ & 6.2 \\
\hline $\mathrm{Bi}$ & $<0.080$ & $<0.080$ & $<0.080$ & $<0.08$ & $<0.080$ & $<0.080$ & $<0.080$ & $<0.08$ \\
\hline Co & $0.23-0.26$ & $0.32-0.56$ & $0.87-1.2$ & 0.45 & $0.22-0.24$ & $0.29-0.48$ & $0.74-1.06$ & 0.39 \\
\hline $\mathrm{Cr}$ & $<1.7$ & $<1.7$ & $<1.7$ & $<1.7$ & $<1.700$ & $<1.700$ & $<1.700$ & $<1.7$ \\
\hline $\mathrm{Cu}$ & $790-860$ & $1000-1300$ & $1870-2130$ & 1190 & $710-790$ & 860-1060 & $1130-1230$ & 950 \\
\hline $\mathrm{Mn}$ & $<1$ & $<1$ & $<1-1.3$ & 1.0 & $<1$ & $<1$ & $1.1-1.2$ & 1.0 \\
\hline Mo & $0.35-0.46$ & $0.54-1.1$ & $1.3-2.0$ & 0.77 & $0.31-0.47$ & $0.63-1.1$ & $1.7-2.1$ & 0.85 \\
\hline Se & $98-110$ & $115-130$ & $140-160$ & 123 & 109-115 & $122-140$ & $145-160$ & 130 \\
\hline $\mathrm{Tl}$ & $0.06-0.07$ & $0.08-0.13$ & $0.15-0.18$ & 0.1 & $0.06-0.07$ & $0.09-0.12$ & $0.14-0.17$ & 0.1 \\
\hline $\mathrm{V}$ & $<0.2$ & $<0.2$ & $<0.2$ & $<0.2$ & $<0.2$ & $<0.2$ & $<0.2$ & $<0.2$ \\
\hline $\mathrm{Zn}$ & $880-955$ & $1020-1220$ & 1300-1450 & 1130 & 910-990 & 1050-1260 & $1350-1460$ & 1150 \\
\hline
\end{tabular}

${ }^{a} \mathrm{GM}$-geometric mean.

\section{Discussion}

The availability of data on baseline levels of a wide range of trace elements in whole blood and blood plasma/serum as reference values in a population is important information for nutritional and clinical monitoring of potential exposures. Due to increased exposure to trace elements including toxic elements, there have been a number of studies to establish blood trace element reference ranges in France, Canada, Korea and Germany $[8,9,18,23]$. These investigations have shown a wide variation of trace elements in human blood. The data of trace element levels in whole blood and blood plasma from various countries are shown in Tables S1 and S2. Therefore, there have been no studies on the analysis of a wide range of trace elements in blood of healthy population in Queensland, Australia.

\subsection{Non-Essential Trace Elements}

Exposure to non-essential trace elements, which have no biological functions in human body and can be toxic such as $\mathrm{Pb}, \mathrm{Hg}$ and $\mathrm{Cd}$, is a health concern in a workplace. Wide variations for non-essential trace elements such as $\mathrm{Ag}(<0.1-0.82 \mu \mathrm{g} / \mathrm{L})$, As $(<0.2-41.6 \mu \mathrm{g} / \mathrm{L})$, $\mathrm{Br}(2820-12,200 \mu \mathrm{g} / \mathrm{L}), \mathrm{Hg}(<0.8-9.3 \mu \mathrm{g} / \mathrm{L})$ and $\mathrm{Pb}(3.8-49.6 \mu \mathrm{g} / \mathrm{L})$ were observed in whole blood in the present study (Table 5). Relatively low levels of $\mathrm{Sb}$ and $\mathrm{Tl}$ were found in blood of Queenslanders (Table 5). Low levels of blood Sb and Tl have been reported for a population in Sweden and Germany [18,24]. As the blood levels of $\mathrm{Bi}, \mathrm{Cr}, \mathrm{U}$ and $\mathrm{V}$ were below the limit of detection of the method the reference values for these elements were not established. The levels of $\mathrm{V}$ in plasma were low $(0.2-0.22 \mu \mathrm{g} / \mathrm{L})$, and low plasma $\mathrm{V}$ levels $(0.06 \pm 0.03 \mu \mathrm{g} / \mathrm{L})$ have also been reported for a healthy urban Italian population [25]. The Ag blood levels $(<0.1-0.82 \mu \mathrm{g} / \mathrm{L})$ were also low and comparable to 
Ag levels $(<0.017-0.4 \mu \mathrm{g} / \mathrm{L})$ of German adults [18]. As for plasma Al levels, two values (22 and $82 \mu \mathrm{g} / \mathrm{L}$ ) were unexpectedly high and when measured at percentile ranges by gender (Table 8), the levels were within percentile ranges reported for Swiss and Italian adults $[25,26]$.

The high level of As ( $42 \mu \mathrm{g} / \mathrm{L})$ was found in one of the females in this study (Table 6). The high As blood levels $(0.9-59.8 \mu \mathrm{g} / \mathrm{L})$ have been shown for a population of Korean women [27]. The elevated levels of As in blood could be potentially attributed to a high intake of seafood and seaweed which can contain As as harmless compounds of arsenobetaine and arsenosugars [11,28]. Several studies have reported the association of elevated levels of As and $\mathrm{Hg}$ in blood and urine with high intake of seafood and fish [29-32]. In this study the blood $\mathrm{Hg}$ levels for men $(<0.8-7.7 \mu \mathrm{g} / \mathrm{L})$ and women $(<0.8-9.3 \mu \mathrm{g} / \mathrm{L})$ were relatively low and comparable to $\mathrm{Hg}$ levels for women $(0.33-11.0 \mu \mathrm{g} / \mathrm{L})$ and men $(0.32-14.5 \mu \mathrm{g} / \mathrm{L})$ in a Finnish population [33]. The blood Hg levels were observed to increase with age, and the over 40 years age group was significantly higher $(p=0.031)$ in mercury than the under 40 years age group (Table 7, Figure S1).

There has been little information on the levels of $\mathrm{Br}$ in human blood, and surveys of trace element levels in many countries have not reported reference values for $\mathrm{Br}$. In this study the blood $\mathrm{Br}$ levels varied widely for men and women, and the overall mean $(4960 \mu \mathrm{g} / \mathrm{L})$ and range $(2820-12,180 \mu \mathrm{g} / \mathrm{L})$ of all sexes were comparable to the blood Br levels (mean: $5300 \mu \mathrm{g} / \mathrm{L}$, range: $2500-11,700 \mu \mathrm{g} / \mathrm{L}$ ) reported in earlier studies in Queensland, Australia [34]. Relatively low Br levels in blood (3200-5600 $\mu \mathrm{g} / \mathrm{L})$ have been reported for 10 healthy adults of North Carolina residents and Chinese adults $(1050-3600 \mu \mathrm{g} / \mathrm{L})[35,36]$. Analysis of $\mathrm{Br}$ in blood is often associated with concern of occupational exposure from brominated hydrocarbons such as halothane and methyl bromide in the workplace, and elevated blood $\mathrm{Br}$ levels greater than $66,000 \mu \mathrm{g} / \mathrm{L}$ have been observed for workers in some environments [37].

As a toxic heavy metal, $\mathrm{Pb}$ exposure is a major concern in the workplace, and its blood levels in a population of high-risk groups are regularly monitored in many countries. $\mathrm{Pb}$ is known to have strong affinity for erythrocytes, therefore its levels in the whole blood are widely used for assessment of health effects and clinical intervention [38,39]. In this study, the blood $\mathrm{Pb}$ levels showed wide variations for males $(4.9-45.0 \mu \mathrm{g} / \mathrm{L})$ and females $(3.8-49.6 \mu \mathrm{g} / \mathrm{L})$ (Table 6) and were comparable to the reference range values reported for healthy adults in USA and France [8,27] (Table S1). Relatively low levels $(<5-25.5 \mu \mathrm{g} / \mathrm{L})$ of blood $\mathrm{Pb}$ have been reported for pregnant women from Western Australia [40], and the levels are consistent with values obtained in this study. The overall mean and reference range values of blood $\mathrm{Pb}$ levels $(13.6 \mu \mathrm{g} / \mathrm{L} ; 3.8-49.6 \mu \mathrm{g} / \mathrm{L})$ in this study were lower than $50 \mu \mathrm{g} / \mathrm{L}(5 \mu \mathrm{g} / \mathrm{dL})$, the recommended threshold for notification and investigation in Queensland population [41]. Air pollution and emission from mining activities and smelters have been shown to contribute to elevated levels of blood $\mathrm{Pb}(19-224 \mu \mathrm{g} / \mathrm{L}$ or 1.9-22.4 $\mu \mathrm{g} / \mathrm{dL}$ ) in Queensland children [13,42]. The blood $\mathrm{Pb}$ levels tend to increase and accumulate with age, and the present study has shown that the age group over 40 years had blood Pb levels significantly higher $(p=0.003)$ than the age group under 40 years (Table 7, Figure S1). Other studies have also shown that blood $\mathrm{Pb}$ concentrations increase with age and tend to be higher in men than women which could be attributed to diet, working and living environments and lifestyle activities [43,44].

\subsection{Essential Trace Elements}

Numerous essential trace element in blood surveys have been carried out in many countries to assess nutritional status which often reflects the dietary patterns and health of the population. These surveys provide important information in assessing deficiency and excess of trace element status in the population and whether appropriate health intervention programs are required. The diseases associated with trace element deficiency such as cardiomyopathy from Se deficiency and hypothyroidism from iodine deficiency have been reported in some countries where appropriate intervention and elimination programs were 
undertaken [45-47]. The physiological mechanisms and the functional roles of essential trace element metabolism in human health outcomes have been widely reviewed $[1,48,49]$. In the present study, the blood levels of essential trace elements of $\mathrm{Co}, \mathrm{Cu}, \mathrm{I}, \mathrm{Mn}, \mathrm{Mo}$, Se and $\mathrm{Zn}$ were found to vary widely. The levels of Co in whole blood $(<0.2-1.1 \mu \mathrm{g} / \mathrm{L})$ were comparable to German adults $(0.04-0.8 \mu \mathrm{g} / \mathrm{L})$ and its levels in plasma $(0.21-1.3 \mu \mathrm{g} / \mathrm{L})$ were relatively higher than that in the Swiss population $(<0.1-0.155 \mu \mathrm{g} / \mathrm{L})[18,26]$. Whole blood or plasma (serum) is widely used for analysis of $\mathrm{Cu}$ and $\mathrm{Zn}$ because their levels in these matrices are generally high and can provide immediate information on health and nutritional status of individuals [50,51]. The blood $\mathrm{Cu}$ levels for females (710-1420 $\mu \mathrm{g} / \mathrm{L})$ and males (650-950 $\mu \mathrm{g} / \mathrm{L})$ in this study were comparable with Serbian and Korean populations [27,52]. The mean values of plasma $\mathrm{Cu}(1240 \mu \mathrm{g} / \mathrm{L})$ and $\mathrm{Zn}(1140 \mu \mathrm{g} / \mathrm{L})$ in females were consistent with mean values of $\mathrm{Cu}(1016 \pm 375 \mu \mathrm{g} / \mathrm{L})$ and $\mathrm{Zn}(896 \pm 163 \mu \mathrm{g} / \mathrm{L})$ reported for other Australian women [53].

As an essential trace element, Se plays important functional roles in antioxidant enzymes of glutathione peroxidases and thioredoxin reductases, and its levels in whole blood and plasma are widely used to assess health and nutritional status of people [54-56]. In this study, the blood Se levels (118-224 $\mu \mathrm{g} / \mathrm{L})$ were comparable to Se levels reported for adults in Brazil (68-245 $\mu \mathrm{g} / \mathrm{L})$ and Germany (85-182 $\mu \mathrm{g} / \mathrm{L})$ [18,57]. The overall mean of Se in whole blood $(141 \mu \mathrm{g} / \mathrm{L}$, Table 5) and plasma $(130 \mu \mathrm{g} / \mathrm{L}$, Table 5) were higher than the mean values (whole blood: $122 \mu \mathrm{g} / \mathrm{L}$; plasma: $102 \mu \mathrm{g} / \mathrm{L}$ ) reported in 2002 survey of the South Australian population [58]. This survey has also shown the decreasing trends from 1977 to 2002 as a result of decreased Se levels in wheat crops, a source of staple food [58]. The mean plasma Se value $(130 \mu \mathrm{g} / \mathrm{L})$ in this study was relatively higher than the mean value $(110 \mu \mathrm{g} / \mathrm{L})$ found in the southern Tasmanian population [59]. The overall plasma Se levels (mean: $130 \mu \mathrm{g} / \mathrm{L}$, range: $82-180 \mu \mathrm{g} / \mathrm{L}$ ) in our study were consistent with Se levels (mean: $85.6 \mu \mathrm{g} / \mathrm{L}$, range: $55.2-173.7 \mu \mathrm{g} / \mathrm{L}$ ) reported in Queensland population from three different regions earlier [15]. Another Queensland study indicated a decline in Se plasma levels with age, which was different from our data showing an increased trend of Se plasma levels with age [14] (Figure S1). The difference in findings of Se status with age could be explained by dietary patterns or/and intakes of vitamin and mineral supplements which have been shown to increase plasma Se levels [15,59]. In the present study, no information was available on dietary patterns and lifestyles of the participants.

There have been few data on the analysis of blood iodine levels in a population [36]. The use of ICP-MS was found to be effective and satisfactory for the blood I analysis in alkali condition. The levels of whole blood I vary widely among the age and gender groups in the Chinese populations ranging from 13.9 to $33.6 \mu \mathrm{g} / \mathrm{L}$ for adolescents and 14.1 to $812 \mu \mathrm{g} / \mathrm{L}$ for adults [36]. In this study, the overall mean $(30.1 \mu \mathrm{g} / \mathrm{L})$ and the range values $(19.5-82.7 \mu \mathrm{g} / \mathrm{L})$ of whole blood I were within the Chinese population range. Another study of the Chinese adults [60] reported the serum I interquartile range levels of $67.9-84.8 \mu \mathrm{g} / \mathrm{L}$ which were closer to the percentile ranges for females (P90-P97.5: 40.6-60.2 $\mu \mathrm{g} / \mathrm{L}$ ) and males (P90-P97.5: 34.7-37.8 $\mu \mathrm{g} / \mathrm{L}$ ) in this study (Table 8).

\section{Conclusions}

The findings of the present study have demonstrated wide variations of trace element levels in whole blood and plasma of Queensland population. Concentrations of some trace elements were below the LOD of the method. Low levels of Bi, Cr, U and V were found in blood samples. Generally, the trace element blood levels reported in this study were comparable with values found in other countries. The study has revealed significant differences of $\mathrm{Cu}, \mathrm{I}, \mathrm{Tl}$ and $\mathrm{Zn}$ in whole blood and $\mathrm{Cu}$ and Se in plasma between men and women and the trend of increased blood $\mathrm{Pb}$, Se and $\mathrm{Zn}$ with age.

There have been few reports on the analysis of trace elements in blood of adult population of Queensland, Australia. The findings in this study have provided information on a wide range of trace elements in blood and plasma which could be used as reference ranges for assessing nutritional and health status of the Queensland population. Unlike the 
monitoring survey programs in USA with the National Health and Nutrition Examination Survey (HNANES) and Europe with EURO-Trace Element Reference Values in Human Tissue (TERVITH), there is no similar program in Australia to monitor wide range of trace elements in blood of a population for all ages from different geographical regions. A recently published preliminary study of two areas in Queensland with no $\mathrm{Pb}$ mines or smelters found low levels of blood $\mathrm{Pb}(4.1-41 \mu \mathrm{g} / \mathrm{L})$ in age groups from 0.5 year to 37 years including pregnant women [61]. Excluding for blood Pb levels, there is little study to include a wide range of other trace element levels in the blood of Queensland population and across Australia, and this should be considered for future study to provide further information on baseline levels of blood trace elements.

Supplementary Materials: The following are available online at https:/ / www.mdpi.com/1660-460 1/18/5/2652/s1, Figure S1: Trends and correlations between trace element levels in blood, plasma and age, Table S1: Concentration of trace elements in human whole blood for both genders by country $[8,18,27,33,44,52,57,62-66]$, Table S2: Concentration of trace elements in human blood plasma for both genders by country $[24,26,27,52,67]$.

Author Contributions: Conceptualization, U.T., T.K. and A.V.P.; Methodology and Validation, T.K.; Statistical and Data Analysis, D.M.; Data evaluation and Writing-Original Draft Preparation, U.T.; Writing-Review and Editing, U.T., T.K., A.V.P. and D.M.; Supervision, U.T.; Project Administration and Funding Acquisition, T.K., U.T., A.V.P. and D.M. All authors have read and agreed to the published version of the manuscript.

Funding: This research was funded by Queensland Health grant No. RSS 19-011.

Institutional Review Board Statement: The study to conduct analysis of human blood was approved by Griffith University (HREC 2016/423) and Mater Medical Research (Mater Misericordia HREC/MML/64703).

Informed Consent Statement: Informed content was organised between the Red Cross Bank and the subjects who voluntarily donated blood in the general public.

Data Availability Statement: The data presented in this study are available on request from the corresponding author. The data are not publicly available due to privacy of internal governmental report.

Acknowledgments: This research was funded by Queensland Health grant RSS 19-011. The funder was not involved in the study design, sample collection, analysis and interpretation of data, or the preparation of the article. We also like to extend our thanks to Ron Sumner for his assistance with ICP-MS analysis.

Conflicts of Interest: The authors declare no conflict of interest.

\section{References}

1. Fraga, C.G. Relevance, essentiality and toxicity of trace elements in human health. Mol. Asp. Med. 2005, 26, 235-244. [CrossRef]

2. Karri, V.; Schuhmacher, M.; Kumar, V. Heavy metals (Pb, Cd, As and MeHg) as risk factors for cognitive dysfunction: A general review of metal mixture mechanism in brain. Environ. Toxicol. Pharmacol. 2016, 48, 203-213. [CrossRef] [PubMed]

3. Schomburg, L.; Köhrle, J. On the importance of selenium and iodine metabolism for thyroid hormone biosynthesis and human health. Mol. Nutr. Food Res. 2008, 52, 1235-1246. [CrossRef] [PubMed]

4. Zimmermann, M.B. The Influence of Iron Status on Iodine Utilization and Thyroid Function. Annu. Rev. Nutr. 2006, 26, 367-389. [CrossRef] [PubMed]

5. Cesbron, A.; Saussereau, E.; Mahieu, L.; Couland, I.; Guerbet, M.; Goullé, J.-P. Metallic Profile of Whole Blood and Plasma in a Series of 106 Healthy Volunteers. J. Anal. Toxicol. 2013, 37, 401-405. [CrossRef] [PubMed]

6. Ivanenko, N.; Ivanenko, A.; Solovyev, N.; Zeimal, A.; Navolotskii, D.; Drobyshev, E. Biomonitoring of 20 trace elements in blood and urine of occupationally exposed workers by sector field inductively coupled plasma mass spectrometry. Talanta 2013, 116, 764-769. [CrossRef] [PubMed]

7. Liu, X.; Zhang, Y.; Piao, J.; Mao, D.; Li, Y.; Li, W.; Yang, L.; Yang, X. Reference Values of 14 Serum Trace Elements for Pregnant Chinese Women: A Cross-Sectional Study in the China Nutrition and Health Survey 2010-2012. Nutrients 2017, 9, 309. [CrossRef] [PubMed]

8. Nisse, C.; Tagne-Fotso, R.; Howsam, M.; Richeval, C.; Labat, L.; Leroyer, A. Blood and urinary levels of metals and metalloids in the general adult population of Northern France: The IMEPOGE study, 2008-2010. Int. J. Hyg. Environ. Health 2017, 220, 341-363. [CrossRef] 
9. Saravanabhavan, G.; Werry, K.; Walker, M.; Haines, D.; Malowany, M.; Khoury, C. Human biomonitoring reference values for metals and trace elements in blood and urine derived from the Canadian Health Measures Survey 2007-2013. Int. J. Hyg. Environ. Health 2017, 220, 189-200. [CrossRef] [PubMed]

10. Wilhelm, M.; Ewers, U.; Schulz, C. Revised and new reference values for some trace elements in blood and urine for human biomonitoring in environmental medicine. Int. J. Hyg. Environ. Health 2004, 207, 69-73. [CrossRef] [PubMed]

11. Davis, M.A.; Gilbert-Diamond, D.; Karagas, M.R.; Li, Z.; Moore, J.H.; Williams, S.M.; Frost, H.R. A Dietary-Wide Association Study (DWAS) of Environmental Metal Exposure in US Children and Adults. PLoS ONE 2014, 9, e104768. [CrossRef] [PubMed]

12. Whitfield, J.B.; Dy, V.; McQUILTY, R.; Zhu, G.; Heath, A.C.; Montgomery, G.W.; Martin, N.G. Genetic Effects on Toxic and Essential Elements in Humans: Arsenic, Cadmium, Copper, Lead, Mercury, Selenium, and Zinc in Erythrocytes. Environ. Health Perspect. 2010, 118, 776-782. [CrossRef]

13. Green, D.; Sullivan, M.; Cooper, N.; Dean, A.; Marquez, C. A Pilot Study of Children's Blood Lead Levels in Mount Isa, Queensland. Int. J. Environ. Res. Public Health 2017, 14, 1567. [CrossRef]

14. Lymbury, R.; Tinggi, U.; Griffiths, L.; Rosenfeldt, F.; Perkins, A.V. Selenium Status of the Australian Population: Effect of Age, Gender and Cardiovascular Disease. Biol. Trace Element Res. 2008, 126, 1-10. [CrossRef] [PubMed]

15. McDonald, C.; Colebourne, K.; Faddy, H.M.; Flower, R.; Fraser, J.F. Plasma selenium status in a group of Australian blood donors and fresh blood components. J. Trace Elements Med. Biol. 2013, 27, 352-354. [CrossRef] [PubMed]

16. Goullé, J.-P.; Mahieu, L.; Castermant, J.; Neveu, N.; Bonneau, L.; Lainé, G.; Bouige, D.; Lacroix, C. Metal and metalloid multielementary ICP-MS validation in whole blood, plasma, urine and hair. Forensic Sci. Int. 2005, 153, 39-44. [CrossRef] [PubMed]

17. Grassin-Delyle, S.; Martin, M.; Hamzaoui, O.; Lamy, E.; Jayle, C.; Sage, E.; Etting, I.; DeVillier, P.; Alvarez, J.-C. A high-resolution ICP-MS method for the determination of 38 inorganic elements in human whole blood, urine, hair and tissues after microwave digestion. Talanta 2019, 199, 228-237. [CrossRef] [PubMed]

18. Heitland, P.; Köster, H.D. Biomonitoring of 37 trace elements in blood samples from inhabitants of northern Germany by ICP-MS J. Trace Elem. Med. Biol. 2006, 20, 253-262. [CrossRef] [PubMed]

19. Jones, D.R.; Jarrett, J.M.; Tevis, D.S.; Franklin, M.; Mullinix, N.J.; Wallon, K.L.; Quarles, C.D.; Caldwell, K.L.; Jones, R.L. Analysis of whole human blood for $\mathrm{Pb}, \mathrm{Cd}, \mathrm{Hg}$, Se, and $\mathrm{Mn}$ by ICP-DRC-MS for biomonitoring and acute exposures. Talanta 2017, 162, 114-122. [CrossRef]

20. Meyer, S.; Markova, M.; Pohl, G.; Marschall, T.A.; Pivovarova, O.; Pfeiffer, A.F.; Schwerdtle, T. Development, validation and application of an ICP-MS/MS method to quantify minerals and (ultra-)trace elements in human serum. J. Trace Elem. Med. Biol. 2018, 49, 157-163. [CrossRef] [PubMed]

21. Gong, Z.-S.; Jiang, X.-H.; Sun, C.-Q.; Tian, Y.-P.; Guo, G.-H.; Zhang, Y.-Z.; Zhao, X.-H.; Wang, Y. Determination of 21 elements in human serum using ICP-MS with collision/reaction cell. Int. J. Mass Spectrom. 2017, 423, 20-26. [CrossRef]

22. ABS. Australian Bureau of Statistics 3101.0-Australian Demographic Statistics. Available online: https://www.abs.gov.au/ ausstats/abs@.nsf/0/1CD2B1952AFC5E7ACA257298000F2E76?OpenDocument (accessed on 14 October 2020).

23. Lee, J.W.; Lee, C.K.; Moon, C.S.; Choi, I.J.; Lee, K.J.; Yi, S.-M.; Jang, B.-K.; Yoon, B.J.; Kim, D.S.; Peak, D.; et al. Korea National Survey for Environmental Pollutants in the Human Body 2008: Heavy metals in the blood or urine of the Korean population. Int. J. Hyg. Environ. Health 2012, 215, 449-457. [CrossRef] [PubMed]

24. Bárány, E.; Bergdahl, I.A.; Bratteby, L.-E.; Lundh, T.; Samuelson, G.; Schütz, A.; Skerfving, S.; Oskarsson, A. Trace element levels in whole blood and serum from Swedish adolescents. Sci. Total. Environ. 2002, 286, 129-141. [CrossRef]

25. Alimonti, A.; Bocca, B.; Mannella, E.; Petrucci, F.; Zennaro, F.; Cotichini, R.; D’Ippolito, C.; Agresti, A.; Caimi, S.; Forte, G. Assessment of reference values for selected elements in a healthy urban population. Ann. dell'Istit. Super. Sanità 2005, 41, 181-187.

26. Forrer, R.; Gautschi, K.; Lutz, H. Simultaneous Measurement of the Trace Elements Al, As, B, Be, Cd, Co, Cu, Fe, Li, Mn, Mo, Ni, $\mathrm{Rb}, \mathrm{Se}, \mathrm{Sr}$, and $\mathrm{Zn}$ in Human Serum and Their Reference Ranges by ICP-MS. Biol. Trace Elem. Res. 2001, 80, 77-93. [CrossRef]

27. Kim, H.-J.; Lim, H.-S.; Lee, K.-R.; Choi, M.-H.; Kang, N.M.; Lee, C.H.; Oh, E.-J.; Park, H.-K. Determination of Trace Metal Levels in the General Population of Korea. Int. J. Environ. Res. Public Health 2017, 14, 702. [CrossRef] [PubMed]

28. Jones, M.R.; Tellez-Plaza, M.; Vaidya, D.; Grau, M.; Francesconi, K.A.; Goessler, W.; Guallar, E.; Post, W.S.; Kaufman, J.D.; Navas-Acien, A. Estimation of Inorganic Arsenic Exposure in Populations With Frequent Seafood Intake: Evidence From MESA and NHANES. Am. J. Epidemiol. 2016, 184, 590-602. [CrossRef] [PubMed]

29. Birgisdottir, B.; Knutsen, H.; Haugen, M.; Gjelstad, I.; Jenssen, M.; Ellingsen, D.; Thomassen, Y.; Alexander, J.; Meltzer, H.; Brantsæter, A. Essential and toxic element concentrations in blood and urine and their associations with diet: Results from a Norwegian population study including high-consumers of seafood and game. Sci. Total Environ. 2013, 836-844. [CrossRef] [PubMed]

30. Karimi, R.; Silbernagel, S.; Fisher, N.S.; Meliker, J.R. Elevated blood Hg at recommended seafood consumption rates in adult seafood consumers. Int. J. Hyg. Environ. Health 2014, 217, 758-764. [CrossRef] [PubMed]

31. Molin, M.; Ulven, S.; Dahl, L.; Goessler, W.; Fliegel, D.; Holck, M.; Sloth, J.J.; Oshaug, A.; Alexander, J.; Meltzer, H.M.; et al. Urinary excretion of arsenicals following daily intake of various seafoods during a two weeks intervention. Food Chem. Toxicol. 2014, 66, 76-88. [CrossRef] [PubMed]

32. Park, S.; Lee, B.-K. Strong Positive Associations Between Seafood, Vegetables, and Alcohol with Blood Mercury and Urinary Arsenic Levels in the Korean Adult Population. Arch. Environ. Contam. Toxicol. 2012, 64, 160-170. [CrossRef] [PubMed] 
33. Abass, K.; Koiranen, M.; Mazej, D.; Tratnik, J.S.; Horvat, M.; Hakkola, J.; Järvelin, M.-R.; Rautio, A. Arsenic, cadmium, lead and mercury levels in blood of Finnish adults and their relation to diet, lifestyle habits and sociodemographic variables. Environ. Sci. Pollut. Res. 2017, 24, 1347-1362. [CrossRef] [PubMed]

34. Olszowy, H.A.; Rossiter, J.; Hegarty, J.; Geoghegan, P.; Haswell-Elkins, M. Background Levels of Bromide in Human Blood. J. Anal. Toxicol. 1998, 22, 225-230. [CrossRef] [PubMed]

35. Cuenca, R.E.; Pories, W.J.; Bray, J. Bromine levels in human serum, urine, hair. Biol. Trace Elem. Res. 1988, 16, 151-154. [CrossRef]

36. Zhang, T.; Wu, Q.; Sun, H.W.; Rao, J.; Kannan, K. Perchlorate and Iodide in Whole Blood Samples from Infants, Children, and Adults in Nanchang, China. Environ. Sci. Technol. 2010, 44, 6947-6953. [CrossRef]

37. Kage, S.; Kudo, K.; Ikeda, H.; Tsujita, A.; Ikeda, N. Determination of bromide in whole blood and urine from humans using gas chromatography-mass spectrometry. J. Chromatogr. B 2005, 817, 335-339. [CrossRef] [PubMed]

38. NHMRC. NHMRC Information Paper: Evidence on the Effects of Lead on Human Health; NHMRC: Canberra, ACT, Australia, 2015; pp. $1-38$.

39. Schultze, B.; Lind, P.M.; Larsson, A.; Lind, L. Whole blood and serum concentrations of metals in a Swedish population-based sample. Scand. J. Clin. Lab. Investig. 2014, 74, 143-148. [CrossRef] [PubMed]

40. Hinwood, A.; Callan, A.; Ramalingam, M.; Boyce, M.; Heyworth, J.; McCafferty, P.; Odland, J. Øyvind Cadmium, lead and mercury exposure in non smoking pregnant women. Environ. Res. 2013, 126, 118-124. [CrossRef] [PubMed]

41. NHMRC. Managing Individual Exposures to Lead in Australia-A Guide for Health Practitioners; National Health and Medical Research Council (NHMRC); NHMRC: Canberra, ACT, Australia, 2016; pp. 1-16.

42. Taylor, M.P.; Schniering, C. The Public Minimization of the Risks Associated With Environmental Lead Exposure and Elevated Blood Lead Levels in Children, Mount Isa, Queensland, Australia. Arch. Environ. Occup. Health 2010, 65, 45-48. [CrossRef]

43. Clark, N.A.; Teschke, K.; Rideout, K.; Copes, R. Trace element levels in adults from the west coast of Canada and associations with age, gender, diet, activities, and levels of other trace elements. Chemosphere 2007, 70, 155-164. [CrossRef] [PubMed]

44. Kim, Y.; Lobdell, D.T.; Wright, C.W.; Gocheva, V.V.; Hudgens, E.; Bowler, R.M. Blood Metal Concentrations of Manganese, Lead, and Cadmium in Relation to Serum Ferritin Levels in Ohio Residents. Biol. Trace Elem. Res. 2015, 165, 1-9. [CrossRef]

45. Chen, J. An original discovery: Selenium deficiency and Keshan disease (an endemic heart disease). Asia Pac. J. Clin. Nutr. 2012, $21,320-326$.

46. Sun, D.; Codling, K.; Chang, S.; Zhang, S.; Shen, H.; Su, X.; Chen, Z.; Scherpbier, R.W.; Yan, J. Eliminating Iodine Deficiency in China: Achievements, Challenges and Global Implications. Nutrients 2017, 9, 361. [CrossRef] [PubMed]

47. Temple, V.; Mapira, P.; Adeniyi, K.; Sims, P. Iodine deficiency in Papua New Guinea (sub-clinical iodine deficiency and salt iodization in the highlands of Papua New Guinea). J. Public Health 2004, 27, 45-48. [CrossRef]

48. McKeating, D.R.; Fisher, J.J.; Perkins, A.V. Elemental Metabolomics and Pregnancy Outcomes. Nutrients 2019, 11, 73. [CrossRef]

49. Zoroddu, M.A.; Aaseth, J.; Crisponi, G.; Medici, S.; Peana, M.; Nurchi, V.M. The essential metals for humans: A brief overview. J. Inorg. Biochem. 2019, 195, 120-129. [CrossRef] [PubMed]

50. Abduljabbar, T.N.; Sharp, B.L.; Reid, H.J.; Barzegar-Befroeid, N.; Peto, T.; Lengyel, I. Determination of Zn, Cu and Fe in human patients' serum using micro-sampling ICP-MS and sample dilution. Talanta 2019, 204, 663-669. [CrossRef]

51. Bocca, B.; Madeddu, R.; Asara, Y.; Tolu, P.; Marchal, J.A.; Forte, G. Assessment of reference ranges for blood Cu, Mn, Se and Zn in a selected Italian population. J. Trace Elem. Med. Biol. 2011, 25, 19-26. [CrossRef]

52. Stojsavljević, A.; Jagodić, J.; Vujotić, L.; Borković-Mitić, S.; Rašić-Milutinović, Z.; Jovanović, D.; Gavrović-Jankulović, M.; Manojlović, D. Reference values for trace essential elements in the whole blood and serum samples of the adult Serbian population: Significance of selenium deficiency. Environ. Sci. Pollut. Res. 2019, 27, 1397-1405. [CrossRef] [PubMed]

53. Fayet-Moore, F.; Petocz, P.; Samman, S. Micronutrient Status in Female University Students: Iron, Zinc, Copper, Selenium, Vitamin B12 and Folate. Nutrients 2014, 6, 5103-5116. [CrossRef] [PubMed]

54. Alfthan, G.; Eurola, M.; Ekholm, P.; Venäläinen, E.-R.; Root, T.; Korkalainen, K.; Hartikainen, H.; Salminen, P.; Hietaniemi, V.; Aspila, P.; et al. Effects of nationwide addition of selenium to fertilizers on foods, and animal and human health in Finland: From deficiency to optimal selenium status of the population. J. Trace Elem. Med. Biol. 2015, 31, 142-147. [CrossRef] [PubMed]

55. Combs, G.F.; Watts, J.C.; Jackson, M.I.; Johnson, L.K.; Zeng, H.; Scheett, A.J.; Uthus, E.O.; Schomburg, L.; Hoeg, A.; Hoefig, C.S.; et al. Determinants of selenium status in healthy adults. Nutr. J. 2011, 10, 1-10. [CrossRef] [PubMed]

56. Tinggi, U. Selenium: Its role as antioxidant in human health. Environ. Health Prev. Med. 2008, 13, 102-108. [CrossRef] [PubMed]

57. Nunes, J.A.; Batista, B.L.; Rodrigues, J.L.; Caldas, N.M.; Neto, J.A.G.; Jr, F.B. A Simple Method Based on ICP-MS for Estimation of Background Levels of Arsenic, Cadmium, Copper, Manganese, Nickel, Lead, and Selenium in Blood of the Brazilian Population. J. Toxicol. Environ. Health Part A 2010, 73, 878-887. [CrossRef]

58. Lyons, G.H.; Judson, G.J.; Stangoulis, J.C.; Palmer, L.T.; Jones, J.A.; Graham, R.D. Trends in selenium status of South Australians. Med. J. Aust. 2004, 180, 383-386. [CrossRef]

59. Jacobson, G.A.; Tong, Y.C.; Townsend, A.T.; Featherstone, A.M.; Ball, M.; Robertson, I.K.; Peterson, G.M. Selenium status in Southern Tasmania. Eur. J. Clin. Nutr. 2007, 61, 1057-1063. [CrossRef] [PubMed]

60. Jin, X.; Jiang, P.; Liu, L.; Jia, Q.; Liu, P.; Meng, F.; Zhang, X.; Guan, Y.; Pang, Y.; Lu, Z.; et al. The application of serum iodine in assessing individual iodine status. Clin. Endocrinol. 2017, 87, 807-814. [CrossRef]

61. Queensland Health. Report: Background Blood Lead Study in Queensland 2018-2019; Queensland Health: Brisbane, QLD, Australia, 2020; pp. 1-14. 
62. Tratnik, J.S.; Mazej, D.; Horvat, M. Analytical Quality Requirements in Human Biomonitoring Programs: Trace Elements in Human Blood. Int. J. Environ. Res. Public Health 2019, 16, 2287. [CrossRef] [PubMed]

63. Zhang, L.L.; Lu, L.; Pan, Y.J.; Ding, C.G.; Xu, D.Y.; Huang, C.F.; Pan, X.F.; Zheng, W. Baseline blood levels of manganese, lead, cadmium, copper, and zinc in residents of Beijing suburb. Environ. Res. 2015, 140, 10-17. [CrossRef] [PubMed]

64. Forte, G.; Madeddu, R.; Tolu, P.; Asara, Y.; Marchal, J.A.; Bocca, B. Reference intervals for blood Cd and Pb in the general population of Sardinia (Italy). Int J. Hyg. Environ. Health 2011, 214, 102-109. [CrossRef] [PubMed]

65. Ikeda, M.; Ohashi, F.; Fukui, Y.; Sakuragi, S.; Moriguchi, J. Cadmium, chromium, lead, manganese and nickel concentrations in blood of women in non-polluted areas in Japan, as determined by inductively coupled plasma-sector field-mass spectrometry. Int. Arch. Occup. Environ. Health 2011, 84, 139-150. [CrossRef] [PubMed]

66. Jain, R.B.; Choi, Y.S. Normal reference ranges for and variability in the levels of blood manganese and selenium by gender, age, and race/ethnicity for general U.S. population. J. Trace Elem. Med. Biol. 2015, 30, 142-152. [CrossRef] [PubMed]

67. Nawi, A.M.; Chin, S.; Jamal, R. Simultaneous analysis of 25 trace elements in micro volume of human serum by inductively coupled plasma mass spectrometry (ICP-MS). Pract. Lab. Med. 2020, 18. [CrossRef] 